Published in final edited form as:

Clin Exp Allergy. 2019 June ; 49(6): 829-837. doi:10.1111/cea.13379.

\title{
Identification of two early life eczema and non-eczema phenotypes with high risk for asthma development
}

\author{
Elisabet Johansson, $\mathrm{PhD}^{\mathrm{a}}$, Jocelyn M. Biagini Myers, $\mathrm{PhD}^{\mathrm{a}}$, Lisa J. Martin, $\mathrm{PhD}^{\mathrm{b}}$, Hua He, \\ MS $^{b}$, Patrick Ryan, $\mathbf{P h D}^{\mathrm{c}}$, Grace K. LeMasters, PhD $^{\mathrm{c}}$, David I. Bernstein, MD ${ }^{\mathrm{d}}$, James \\ Lockey, MD $^{\mathrm{C}}$, and Gurjit K. Khurana Hershey, MD, PhD ${ }^{\mathrm{a}}$ \\ aDivision of Asthma Research, University of Cincinnati, Cincinnati, OH, USA \\ bDivision of Human Genetics, Cincinnati Children's Hospital Medical Center, University of \\ Cincinnati, Cincinnati, OH, USA \\ 'Department of Pediatrics and Department of Environmental Health and University of Cincinnati, \\ Cincinnati, OH, USA \\ dDepartment of Pediatrics Department of Internal Medicine, University of Cincinnati, Cincinnati, \\ $\mathrm{OH}, \mathrm{USA}$
}

\section{Abstract}

Background.-The "atopic march" has been considered a linear progression starting with eczema and culminating with development of asthma. Not all asthma cases, however, are preceded by eczema, and not all children with eczema go on to develop asthma.

Objective.-The aim of this study was to explore the impact of allergic sensitization patterns on the association between early eczema and later childhood asthma. Given the numerous reported associations of the ciliary gene $K I F 3 A$ with the atopic march, we also examined the impact of $K I F 3 A$ risk allele $r s 12186803$ on our analyses.

Methods.-We studied 505 participants in the Cincinnati Childhood Allergy and Air Pollution Study (CCAAPS), a prospective birth cohort, with longitudinal eczema and asthma outcomes as well as prospective data regarding timing of sensitization to foods and aeroallergens. KIF3A genotypes were available on all children.

Results.-Two high-risk groups were identified: one with and one without early eczema. The high-risk group with early eczema was more likely to be sensitized to food allergens, while the group without early eczema was more likely to be poly-sensitized to aeroallergens. The KIF3A rs 12186803 risk allele interacted with food sensitization to increase asthma risk in children with eczema ( $\mathrm{p}=0.02)$. In children without eczema, asthma was associated with the interaction between rs 12186803 and aeroallergen sensitization $(\mathrm{p}=0.007)$.

Conclusions \& Clinical Relevance.-KIF3A interacted differentially with sensitization pattern to increase the risk of asthma in two high-risk groups of children with and without early

Corresponding author: Gurjit K. Khurana Hershey, Division of Asthma Research, Cincinnati Children's Hospital Medical Center, Cincinnati, OH 45229. Gurjit.Hershey@ cchmc.org, Tel: (513)636-7054, Fax: (513)636-1657.

Conflict of interest: The authors have declared that there are no conflicts of interest. 
eczema. Given the reported role of KIF3A in epithelial cell functioning, the results adds evidence to the hypothesis that an impaired epithelial barrier is a key aspect in the development of allergic disease.

\section{Keywords}

eczema; atopic dermatitis; asthma; phenotype; KIF3A

\section{Introduction}

The concept of the atopic march is based on observations that eczema tends to precede later development of allergic rhinitis and asthma. The causal nature of this progression, however, remains a topic of considerable debate. The atopic march concept postulates that eczema precedes and promotes the development of food and airway allergies[1]. Indeed, it is well established that eczema in early childhood is associated with an increased risk of later asthma, but estimates of the asthma risk among children with eczema vary greatly[1,2]. Although eczema is a major risk factor for later airway allergies[1,3], only a fraction of children with early eczema go on to develop asthma. Furthermore, not all asthma cases are preceded by eczema[4]. In the Tucson Children's Respiratory Study, only $18 \%$ of children with wheezing at age 6 had eczema before age 2[5]. Thus, progression from eczema to asthma represents only one pathway of asthma development [6, 7], and the "atopic march" may not represent the most common phenotype of disease progression to asthma.

Another well recognized risk factor for asthma is allergic sensitization[3, 8]. Evidence suggests that the risk for asthma may be related to the number and type of sensitizing allergens as well as the timing of sensitization[9, 10]. Early aeroallergen sensitization (AS), especially to multiple aeroallergens, increases asthma risk in school-age children[11-13]. Food sensitization (FS) in early childhood, as well as co-sensitization to both food and aeroallergens $(\mathrm{CoS})$, has also been associated with asthma[14, 15]. Additionally, a faulty skin barrier and/or early eczema is associated with FS[16].

The challenge is that the risk of asthma seems to be due to combinations of factors rather than any one factor such as eczema or allergic sensitization. One possible factor, which may further modify risk, is underlying genetic variation such as Kinesin family member $3 \mathrm{~A}$ (KIF3A). KIF3A is a component of both primary and motile cilia, has been consistently associated with asthma and eczema[17-22]. More recently, a KIF3A genotype has been associated with eczema and asthma co-morbidity and with the atopic march in a genome wide association study[23, 24]. In animal models, deletion of Kif3a in epidermal cells results in disrupted keratinocyte differentiation[25, 26], suggesting a role for KIF3A in skin barrier function. Deletion of Kif3a in mouse airway epithelial cells resulted in enhanced asthma development including increased airway hyperreponsiveness and airway inflammation. Mice deficient for Kif3a in the airways epithelium had impaired airway epithelial barrier function and impaired mucociliary clearance[27]. Thus, KIF3A plays a critical role in epithelial barrier function in several target organs and may modify asthma risk for eczema and allergic sensitization. 
Based on the prior work, we hypothesized that eczema and allergic sentization may act in isolation or jointly, and that their effects may be modified by underlying $K I F 3 A$ genetic variation. To test this hypothesis we first evaluated the relationship between timing and patterns of allergic sensitization with asthma and eczema separately in a prospective birth cohort. We then evaluated relationship specific allergic sensitization patterns and asthma stratified by eczema. Herein, we investigate the interplay between the timing and pattern of allergic sensitization and eczema in conferring asthma risk in a prospective birth cohort. We then evaluate whether KIF3A genetic vairaiton modifes these relationships. This systematic approach allowed use to generate models which were informed by both our results as well as prior studies.

\section{Methods}

\section{Study cohort}

The Cincinnati Childhood Allergy and Air Pollution Study (CCAAPS) has been described in detail previously[28]. Briefly, CCAAPS is a birth cohort of 762 infants born to atopic parent(s) between 2001 and 2003. Children were examined at 1, 2, 3, 4 and 7 years of age for the development of allergic disease. Informed consent was obtained from a parent, and the study was approved by the Institutional Review Board at the University of Cincinnati and Cincinnati Children"s.

The presence of eczema was evaluated at each visit by a study physician as previously described[29]. Eczema diagnosis was based on a global assessment measure, and findings considered to be consistent with eczema included erythema, papulation, excoriations, or lichenification. Early eczema was defined as an eczema diagnosis at ages 1, 2, or 3 years. Pulmonary function was assessed at age 7 and asthma was diagnosed according to ATS-ERS guidelines[30]. Early wheezing was defined as $\geq 1$ parent-reported wheezing episodes not associated with, and explained by, the presence of cold at ages 1, 2, or 3 years.

At each visit, CCAAPS children underwent skin prick testing (SPT) to milk, egg, and 15 aeroallergens: (meadow fescue, timothy, short ragweed, white oak, maple mix, American elm, red cedar, Alternaria, Aspergillus fumigatus, Penicillium mix, Cladosporium, cat, dog, German cockroach [Blattella germanica] and dust mite mix [Dermatophagoides farinae and Dermatophagoides pteronyssinus])[28]. A positive SPT was defined as a wheal $>3 \mathrm{~mm}$ greater than the negative control. Sensitization patterns are defined in Table 1. Polysensitization is defined as sensitization to 2 or more allergens. Because CCAAPS children were skin prick tested to only 2 foods, we did not have sufficient information to examine polysensitization to foods. Early sensitization was defined as sensitization at ages 1, 2 , or 3 years.

\section{Genotyping of KIF3A}

In a previous study, genotyping for single nucleotide polymorphisms (SNPs) in KIF3A had been done for 504 CCAAPS children[24]. In that study, rs 12186803 was the most strongly associated with the asthma+eczema phenotype. Therefore, we focused our analyses on this 
SNP. In this study, the risk allele was defined as the rs12186803 variant associated with higher risk of asthma in previous studies (the A allele)[20, 24].

\section{Statistical analysis}

Five hundred and eighty-nine CCAAPS children were tested for asthma at age 7, and 490 of these children were evaluated for allergic sensitization and eczema in the first 3 years of life, and had KIF3A rs 12186803 genotype data available. Supplemental figure 1 describes how the study population was obtained. Among the children without any positive test for any of the first three years, children who tested negative for AS or FS, respectively, for at least one of years 1, 2, or 3 were retained in the study and defined as AS or FS controls even if information was missing for one or two years (Supplemental table 1). This was done in order to maintain an adequate sample size, Likewise, children with a negative eczema diagnosis for at least one of years 1, 2, or 3 defined as eczema controls. The rs 12186803 SNP passed the Hardy-Weinberg equilibrium test $(\mathrm{p}=0.14)$. The demographic and clinical characteristics were compared between asthma and no asthma using chi-square test. T-test was performed to compare age of first AS in children sensitized vs. not sensitized to food allergens by year 1.

Unadjusted logistic regression was performed to assess the association of asthma with different sensitization patterns. Multiple logistic regression model was also performed to examine the association of asthma with aerosensitization and food sensitization after adjusting for other factors which have been associated with asthma (race, wheezing in the absence of cold symptoms, and parental asthma). An additive model was applied for the rs $12186803 \mathrm{SNP}$ in the multiple regression analyses. In the regression models, both FS and AS were modeled as continuous variables based on the number of sensitizations. The interactions of $K I F 3 A$ rs 12186803 genotype with AS and FS respectively were also tested in logistic regression models, with race, early wheezing in the absence of cold symptoms, and parental asthma included as covariates. All the logistic regression analysis were performed in children with and without eczema. To further explore the association of asthma with $K I F 3 A$ and FS in early eczema kids, a 4-level variable ( $K I F 3 A-$, FS-; $K I F 3 A+$, FS-; $K I F 3 A-, \mathrm{FS}+$; $K I F 3 A+, \mathrm{FS}+$ ) was created, which was adjusted for early wheezing and parental asthma. KIF3A+ indicates having at least one risk $r s 12186803 \mathrm{~A}$ allele of $K I F 3 A$, and $K I F 3 A$ - indicates having no copies of the risk $r s 12186803$ A allele. Similarly, another 4level variable, which was adjusted for race and early wheezing, was created to further investigate the effect of $K I F 3 A$ and poly-AS on asthma in children without early eczema.

In all analyses, a p-value $<0.05$ was considered statistically significant.

\section{Results}

\section{Characteristics of the cohort}

Table 2 displays the demographic and clinical characteristics, stratified by asthma diagnosis. The study cohort was 21\% African American and 58\% male, similar to what has previously been published for CCAAPS[31]. Parental asthma was reported for $40.6 \%$ of study participants, and $17.6 \%$ had a history of early wheeze. At age $7,17 \%$ of the children were 
diagnosed with asthma (Table 2). African-American children were more likely to be asthmatics (38\% vs. 17\%, p<0.001). There were no differences in sex (Table 2).

\section{Sensitization patterns in CCAAPS children}

SPT patterns are shown in Table 3. Early positive SPT was found in $61 \%$ of children, and $79 \%$ of children had positive SPT by age 7 years. Fifty-seven percent of children had early AS (aeroallergen sensitization), and 19\% had early FS (food sensitization). At age 7, 77\% had AS and $21 \%$ had FS. Of the children with early positive poly-SPT, $64 \%$ were polysensitized to aeroallergens only, 35\% had $\mathrm{CoS}$ (co-sensitization to both food and aeroallergens) to at least one food and one aeroallergen, and $1 \%$ were polysensitized to food allergens only.

\section{Timing of sensitization is associated with asthma outcome at age 7}

Among asthmatics and non-asthmatics, $73 \%$ and $54 \%(\mathrm{OR}=2.3 ; \mathrm{p}=0.002)$ had early AS, respectively, while $85 \%$ and $76 \%$, respectively had AS by age $7(\mathrm{OR}=1.8 ; \mathrm{p}=0.10)$ (Table 3$)$. Thus, asthma at age 7 was associated with early AS, but not AS through age seven. Further stratification of early AS into mono-AS and poly-AS showed that the association between asthma and early AS was mainly driven by early poly-AS, which was significantly associated with asthma $(\mathrm{OR}=2.6 ; \mathrm{p}=0.001)$ whereas mono-AS was not.

In terms of FS, $28 \%$ and $17 \%$ of children with and without asthma by age 7, respectively, had early FS (OR=1.9; $\mathrm{p}=0.03$ ), while $29 \%$ and $19 \%$, respectively, had FS by age seven $(\mathrm{OR}=1.8 ; \mathrm{p}=0.04)$. Since few participants were sensitized to both egg and milk, it was not possible to determine the respective contributions of mono-FS vs. poly-FS with asthma. Thus, we focused our further analyses on early FS and AS.

\section{Sensitization load is associated with asthma at age 7}

The rate of early positive poly-SPT among children was $72 \%$ for those with asthma and $48 \%$ without asthma at age 7 years $(\mathrm{OR}=2.7 ; \mathrm{p}=0.0005$, Table 3$)$. Asthmatics were more likely to be $\operatorname{CoS}(25 \%$ vs. $13 \% \mathrm{CoS}$ in non-asthmatics, $\mathrm{OR}=2.2 ; \mathrm{p}=0.009)$. Similarly, $38 \%$ vs. $59 \%$ of children without and with asthma, had early poly-AS only $(\mathrm{OR}=2.3 ; \mathrm{p}=0.004)$. Thus, the total load of sensitization is associated with asthma, and this was evident in children with early poly-AS and CoS.

With respect to timing of FS and AS among those with $\mathrm{CoS}$ (through age seven; N=92); $70 \%$ were FS by their first birthday; FS preceded AS in 52\% of CoS children, AS preceded FS in 17\%, and $31 \%$ became CoS in the same year (Figure 1A). Wilcoxon rank sum test detected a significant shift in age of first AS for children FS vs not FS by year $1(\mathrm{p}=0.001$; Median (interquartile range) $=2(1,3)$ vs. $2(2,4)$ )

\section{Associations between eczema and patterns of sensitization}

By age 3,36.4\% of children had eczema (eczema by age 3 defined as early eczema) (Table 2). Eczema was associated with both early AS and early FS (Figure 1B). Among children with eczema by age 3,67\% had early AS, while $51 \%$ of children without eczema by age 3 had early AS (Supplemental table 2; $\mathrm{p}=0.001$ ). In terms of FS, $31 \%$ with eczema by age 3 
had FS, while $13 \%$ of children without eczema by age 3 had early FS ( $p<0.0001$ ).

Similarly, CoS to both food and aeroallergens by age 3 was more common in children with $(25 \%)$ versus without $(9 \%)$ early eczema $(\mathrm{p}<0.0001)$. Early poly-AS was also more prevalent among children with vs. without eczema (50\% vs. 30\%; p<0.001). The odds ratios for FS, AS, poly-AS, and $\mathrm{CoS}$ in the presence of early eczema were $3.0(1.9,4.9), 1.9(1.3$, 2.8), $2.4(1.6,3.7)$, and $3.3(2.0,5.5)$, respectively. Although eczema was associated with an increased risk of AS and poly-AS, the odds ratios were lower compared to those for FS in the presence of early eczema. Among children with AS, 58\% did not have eczema, and among those with poly-AS, 52\% did not have eczema. In contrast, only $43 \%$ with FS did not have eczema and $39 \%$ with CoS did not have eczema. Also, the presence or absence of eczema did not alter the mean age of AS sensitization in children with FS compared to those without FS (data not shown). Collectively, these data suggest that while eczema is associated with AS and FS, significant sensitization occurs in the absence of eczema, and these children may also be at high risk for asthma.

\section{Asthma risk as a function of eczema and sensitization pattern}

As expected, a history of early eczema was more common in children with asthma at age 7 $(47 \%)$ compared to non-asthmatics $(34 \%),(\mathrm{p}=0.03)$. We next examined the prevalence of asthma for each sensitization pattern, stratified by early eczema (Figures 1C and1D and Supplemental Table 3). Overall, asthma prevalence rates were increased in sensitized children regardless of the type of sensitization. In children without a history of eczema by age 3 years, the odds ratios for asthma prevalence in the absence vs. presence of sensitization were significant for all AS patterns. In contrast, FS was not associated with asthma. These results suggest that AS, but not FS, at least to milk and eggs, is a driver of asthma risk in children without early eczema. Although asthma prevalence trended higher in the presence vs. absence of sensitization among children with a history of eczema by age 3 years, the difference was not significant for any of the sensitization patterns analyzed. Thus, $\mathrm{AS}$ is associated with asthma risk among children without eczema, while eczema is an independent risk factor for asthma regardless of sensitization.

\section{Regression analysis of asthma risk as a function of sensitization load in the presence vs. absence of eczema}

In a multivariate model where FS and AS variables were modeled as continuous variables, AS load, but not FS load, was associated with asthma among children without a history of early eczema ( $\mathrm{p}=0.02$ vs. $\mathrm{p}=0.2$ ) (Table $4 \mathrm{~A}$ ). Among children with early eczema, neither AS nor FS was significantly associated with asthma risk. Interestingly, being African-American was associated with asthma risk among children without early eczema $(p=0.005)$, but not among those with early eczema. In contrast, parental asthma was only associated with asthma in children with a history of early eczema $(\mathrm{p}=0.02)$.

\section{KIF3A rs12186803 genotype confers risk for FS in children with early eczema}

The presence of one or two rs 12186803 risk alleles was significantly associated with early FS (OR=1.7; $\mathrm{p}=0.02$ ) (Figure 2A), whereas no association with early AS was found.

Stratification for eczema showed that the presence of one or two rs 12186803 risk alleles was associated with a higher risk of early FS, but only in children with early eczema ( $\mathrm{OR}=2.0$; 
$\mathrm{p}=0.04$ ) (Figure 2B). In contrast, KIF3A rs 12186803 genotype was not associated with early AS regardless of eczema (Figure $2 \mathrm{C}$ ).

\section{KIF3A rs12186803 genotype and early FS define a group with high risk of asthma in children with early eczema}

To further explore the impact of early FS or AS sensitization and KIF3A rs 12186803 genotype on asthma outcome among children with early eczema, we performed regression analyses (FS and AS were again modeled as continuous variables). In children with early eczema, KIF3A rs 12186803 genotype and FS interacted to significantly increase the risk of asthma at age seven $(\mathrm{p}=0.02)$, although neither variable on its own was associated with asthma risk (Table 4B). No similar interaction effect was observed for early AS. The interaction effect between KIF3A rs 12186803 genotype and early FS was not affected by inclusion of early AS in the model. There were too few children with FS without AS ( $n=22)$; thus we were not powered to examine this subgroup independently.

A forest plot was constructed to show how KIF3A rs 12186803 genotype and early FS individually and in combination are associated with asthma risk in children with early eczema (Figure 3A). Whereas children with both the KIF3A rs 12186803 risk genotype and early FS had a significantly increased risk of asthma, neither KIF3A rs 12186803 genotype nor early FS alone was associated with asthma risk. The results demonstrate that the association between KIF3A rs 12186803 genotype and progression to asthma in children with eczema (the atopic march-like phenotype) is modified by early FS.

\section{In children without early eczema, the association between KIF3A rs12186803 genotype and asthma risk is modified by early poly-AS}

In contrast to our above observations in children with early eczema (the atopic march-like phenotype), we found no interaction between early FS and KIF3A rs 12186803 genotype in children without eczema. Early poly-AS, however, interacted with KIF3A rs 12186803 genotype to increase the risk of asthma at age 7 in children without eczema ( $\mathrm{p}=0.007)$ (Table 4B). As in the regression models without KIF3A rs 12186803 genotype included, in children without early eczema, being African American was associated with increased asthma risk $(p=0.002)$, an effect not observed in children with preceding early eczema.

To explore the combinatorial effects of KIF3A genotype and early AS in children without early eczema, a forest plot was created (Figure 3B). Since African-American race was associated with asthma, the forest plot was adjusted for race. Having both the risk KIF3A rs 12186803 genotype and early poly-AS was associated with an increased odds ratio for asthma risk $(4.1 ; 1.7,10.2)$ relative to children neither risk KIF3A rs 12186803 genotype nor poly-AS. A slightly increased odds ratio was seen for poly-AS in the absence of the risk KIF3A rs 12186803 genotype.

\section{Discussion}

We identified two distinct groups of young children who are at high risk for the development of asthma, schematically outlined in Figure 4. One of the high-risk groups had eczema, while the other did not. Both groups had early allergic sensitization by age 3, but they had 
different patterns of sensitization. The high-risk group with eczema was more likely to be sensitized to food allergens, while the high-risk group without eczema was more likely to be poly-sensitized to aeroallergens. The KIF3A rs 12186803 risk allele differentially interacted with the sensitization pattern to modify asthma risk in both groups, resulting in high risk for asthma even without clinical eczema. As such, the atopic march only represents one asthma phenotype. This is highly relevant as eczema is one of the major criteria of the Asthma Predictive Index $[32,33]$. The fact is that a large number of children develop asthma without ever having been diagnosed with eczema[6,34], and these children are often marginalized due to the focus on the atopic march. As such, these children are often not prioritized to populate asthma prevention studies, which utilize the Asthma Predictive Index to identify children for inclusion[35, 36].

An important observation is that asthma risk in both of the high-risk groups was modified by genetic variation in $K I F 3 A$, which has been reported by numerous GWAS and genetic association studies to be associated with eczema, asthma, and the atopic march[17-24]. The KIF3A rs 12186803 risk allele differentially interacted with the distinct sensitization pattern in each high-risk group to modify asthma risk. In children with eczema, KIF3A rs 12186803 genotype interacted with early FS to define a group with high risk of asthma, while in children without eczema, KIF3A rs 12186803 genotype interacted with early poly-AS to define a group with high risk of asthma. Coding for a subunit of the kinesin-2 motor complex, KIF3A is a pleiotropic gene that play a role in the formation and/or functioning of primary and motile cilia[37]. Deletion of Kif3a in the epidermis of mice results in dysregulated keratinocyte proliferation and differentiation[25, 26], whereas Kif3a deletion in airway epithelial cells results in impaired barrier function in the airways[27]. Thus, KIF3A impacts both the skin and lung barriers. In the presence of eczema, it may promote sensitization through the skin to food allergens; and in the absence of eczema, it may promote sensitization to inhaled allergens. Our finding that $K I F 3 A$ variation is associated with asthma is consistent with previous reports[20-22]. Further, eczema strengthening this association has also been reported[23, 24]. Our finding that $K I F 3 A$ modifies the association between eczema and FS in the context of eczema is novel. This suggests that the initial entry point for food sensitization may be the skin in the context of a compromised skin barrier as is the case in eczema[38]. Indeed, our work is consistent with prior studies for the skin barrier gene filaggrin $(F L G)$. Loss of function mutations in $F L G$ are associated with FS and food allergy in early childhood[39, 40]. The reason that an impaired skin barrier appear to be associated specifically with early FS and not AS will have to be addressed in future studies. It may be a question of different exposure routes for food versus aeroallergens, in that infants and small children may be more likely to be exposed topically to food, whereas aeroallergens are usually inhaled.

The second high-risk group characterized by poly-sensitization to aeroallergens in the absence of early eczema, is an important high-risk group. We hypothesize that while these children do not exhibit eczema, they may have epithelial barrier defects at the skin or lung mucosal surfaces that do not manifest as clinical eczema. Further, we found that in children without early eczema, being African-American was a predictor of asthma risk $(\mathrm{p}=0.012)$. These data suggest that this second high risk group may be more relevant to the AfricanAmerican population. In fact, among African-American children in our study who were 
diagnosed with asthma, a majority lacked a history of early eczema. African-American children face significant morbidity and mortality from asthma[41], and it is critical that this population and high risk group be better recognized in future studies, especially intervention and prevention trials.

A major strength of our study is the prospective longitudinal CCAAPS birth cohort, which had extensive careful phenotypic and clinical data as well as genotype data available. However, there were also some important limitations. The strong association between FS and AS lead to a high degree of CoA, and only $3 \%$ of the children were sensitized to food only. Thus, we could not examine the impact of FS without AS. Our regression models suggested that the interaction between KIF3A rs 12186803 genotype and FS was independent of AS, but this remains to be determined. Further, since children in the CCAAPS cohort were only tested for two food allergens, milk and egg, some of the children classified as FS-negative may have been sensitized to other food allergens that were not examined, such as peanut. However, egg is the most common early food allergy in the general population, with an estimated prevalence of $1.6 \%$ among unselected children aged between 2.5 and 3 years[42, 43]. Finally, the definition of early sensitization controls as having a negative SPT test for at least one of years 1, 2, or 3 may have resulted in misclassification of some cases as controls. However, in over $85 \%$ of the study population, AS information was available for all 3 years, and the same was the case for FS.

In summary, the atopic march only represents one phenotype of asthma, and there is another early life high-risk group for asthma of children with early poly-AS without eczema. This group may be overrepresented in the African-American population, and has been often marginalized with the focus on the atopic march phenotype. Interestingly, asthma risk in both groups was modified by the $K I F 3 A$ risk allele.

\section{Supplementary Material}

Refer to Web version on PubMed Central for supplementary material.

\section{Acknowledgments}

Funding Source:

Supported by NIH U19 AI1070235 (GKKH)

\section{Abbreviations:}

TEWL transepidermal water loss

KIF3A Kinesin family member 3A

AS aeroallergen sensitization

FS food sensitization

CoS co-sensitization to both food and aeroallergens

CCAAPS Cincinnati Childhood Allergy and Air Pollution Study 

FLG
filaggrin gene
SNP
single nucleotide polymorphism
GWAS
genome-wide association study

\section{REFERENCES}

1. Bantz SK, Zhu Z, and Zheng T, The Atopic March: Progression from Atopic Dermatitis to Allergic Rhinitis and Asthma. J Clin Cell Immunol, 2014 5(2).

2. van der Hulst AE, Klip H, and Brand PL, Risk of developing asthma in young children with atopic eczema: a systematic review. J Allergy Clin Immunol, 2007 120(3): p. 565-9. [PubMed: 17655920]

3. Amat F, et al., Early-Onset Atopic Dermatitis in Children: Which Are the Phenotypes at Risk of Asthma? Results from the ORCA Cohort. PLoS One, 2015 10(6): p. e0131369. [PubMed: 26107938]

4. Rerknimitr P, et al., The etiopathogenesis of atopic dermatitis: barrier disruption, immunological derangement, and pruritus. Inflamm Regen, 2017 37: p. 14. [PubMed: 29259713]

5. Martinez FD, et al., Asthma and wheezing in the first six years of life. The Group Health Medical Associates. N Engl J Med, 1995 332(3): p. 133-8. [PubMed: 7800004]

6. Belgrave DC, et al., Developmental profiles of eczema, wheeze, and rhinitis: two population-based birth cohort studies. PLoS Med, 2014 11(10): p. e1001748. [PubMed: 25335105]

7. Panico L, et al., Asthma trajectories in early childhood: identifying modifiable factors. PLoS One, 2014 9(11): p. e111922. [PubMed: 25379671]

8. Wisniewski JA, et al., Sensitization to food and inhalant allergens in relation to age and wheeze among children with atopic dermatitis. Clin Exp Allergy, 2013 43(10): p. 1160-70. [PubMed: 24074334]

9. Alduraywish SA, et al., The march from early life food sensitization to allergic disease: a systematic review and meta-analyses of birth cohort studies. Allergy, 2016 71(1): p. 77-89. [PubMed: 26466117]

10. Moustaki M, et al., The Role of Sensitization to Allergen in Asthma Prediction and Prevention. Front Pediatr, 2017 5: p. 166. [PubMed: 28824890]

11. Amin P, et al., Optimum predictors of childhood asthma: persistent wheeze or the Asthma Predictive Index? J Allergy Clin Immunol Pract, 2014 2(6): p. 709-15. [PubMed: 25439361]

12. Calamelli E, et al., Patterns of aeroallergen sensitization predicting risk for asthma in preschool children with atopic dermatitis. J Asthma, 2015 52(5): p. 458-64. [PubMed: 25387149]

13. Stoltz DJ, et al., Specific patterns of allergic sensitization in early childhood and asthma \& rhinitis risk. Clin Exp Allergy, 2013 43(2): p. 233-41. [PubMed: 23331564]

14. Kjaer HF, et al., The association between early sensitization patterns and subsequent allergic disease. The DARC birth cohort study. Pediatr Allergy Immunol, 2009 20(8): p. 726-34. [PubMed: 19744222]

15. Alduraywish SA, et al., Is there a march from early food sensitization to later childhood allergic airway disease? Results from two prospective birth cohort studies. Pediatr Allergy Immunol, 2017 28(1): p. 30-37. [PubMed: 27590890]

16. Tsakok T, et al., Does atopic dermatitis cause food allergy? A systematic review. J Allergy Clin Immunol, 2016 137(4): p. 1071-8. [PubMed: 26897122]

17. Lepre T, et al., Association of KIF3A, but not OVOL1 and ACTL9, with atopic eczema in Italian patients. Br J Dermatol, 2013 168(5): p. 1106-8. [PubMed: 23278845]

18. Hirota T, et al., Genome-wide association study identifies eight new susceptibility loci for atopic dermatitis in the Japanese population. Nat Genet, 2012 44(11): p. 1222-6. [PubMed: 23042114]

19. Paternoster L, et al., Meta-analysis of genome-wide association studies identifies three new risk loci for atopic dermatitis. Nat Genet, 2012 44(2): p. 187-92. 
20. Butsch Kovacic M., et al., Identification of KIF3A as a Novel Candidate Gene for Childhood Asthma Using RNA Expression and Population Allelic Frequencies Differences. PLoS One, 2011 6(8): p. e23714. [PubMed: 21912604]

21. Kim JH, et al., KIF3A, a cilia structural gene on chromosome $5 q 31$, and its polymorphisms show an association with aspirin hypersensitivity in asthma. J Clin Immunol, 2011 31(1): p. 112-21. [PubMed: 20922562]

22. Michel S, et al., Unifying candidate gene and GWAS Approaches in Asthma. PLoS One. 5(11): p. e13894.

23. Marenholz I, et al., Meta-analysis identifies seven susceptibility loci involved in the atopic march. Nat Commun, 2015 6: p. 8804. [PubMed: 26542096]

24. Johansson E, et al., KIF3A genetic variation is associated with pediatric asthma in the presence of eczema independent of allergic rhinitis. J Allergy Clin Immunol, 2017 140(2): p. 595-598 e5. [PubMed: 28238750]

25. Ezratty EJ, et al., A role for the primary cilium in Notch signaling and epidermal differentiation during skin development. Cell, 2011 145(7): p. 1129-41. [PubMed: 21703454]

26. Croyle MJ, et al., Role of epidermal primary cilia in the homeostasis of skin and hair follicles. Development, 2011 138(9): p. 1675-85. [PubMed: 21429982]

27. Giridhar PV, et al., Airway Epithelial KIF3A Regulates Th2 Responses to Aeroallergens. J Immunol, 2016 197(11): p. 4228-4239. [PubMed: 27794000]

28. LeMasters GK, et al., High prevalence of aeroallergen sensitization among infants of atopic parents. J Pediatr, 2006 149(4): p. 505-11. [PubMed: 17011322]

29. Epstein TG, et al., Opposing effects of cat and dog ownership and allergic sensitization on eczema in an atopic birth cohort. J Pediatr, 2011 158(2): p. 265-71 e1-5. [PubMed: 20884006]

30. Reponen T, et al., Infant origins of childhood asthma associated with specific molds. J Allergy Clin Immunol, 2012 130(3): p. 639-644 e5. [PubMed: 22789397]

31. Biagini JM, et al., Environmental risk factors of rhinitis in early infancy. Pediatr Allergy Immunol, 2006 17(4): p. 278-84. [PubMed: 16771781]

32. Castro-Rodriguez JA, et al., A clinical index to define risk of asthma in young children with recurrent wheezing. Am J Respir Crit Care Med, 2000 162(4 Pt 1): p. 1403-6. [PubMed: 11029352]

33. Chang TS, et al., Evaluation of the modified asthma predictive index in high-risk preschool children. J Allergy Clin Immunol Pract, 2013 1(2): p. 152-6. [PubMed: 24187656]

34. Hopper JL, et al., Does eczema in infancy cause hay fever, asthma, or both in childhood? Insights from a novel regression model of sibling data. J Allergy Clin Immunol, 2012 130(5): p. 1117-1122 e1. [PubMed: 23021888]

35. Lowe AJ, et al., The skin as a target for prevention of the atopic march. Ann Allergy Asthma Immunol, 2018 120(2): p. 145-151. [PubMed: 29413338]

36. Guilbert TW, et al., Long-term inhaled corticosteroids in preschool children at high risk for asthma. N Engl J Med, 2006 354(19): p. 1985-97. [PubMed: 16687711]

37. Malicki J, Who drives the ciliary highway? Bioarchitecture, 2012 2(4): p. 111-7. [PubMed: 22960672]

38. Gupta J, et al., Intrinsically defective skin barrier function in children with atopic dermatitis correlates with disease severity. J Allergy Clin Immunol, 2008 121(3): p. 725-730 e2. [PubMed: 18249438]

39. van Ginkel CD, et al., Loss-of-function variants of the filaggrin gene are associated with clinical reactivity to foods. Allergy, 2015 70(4): p. 461-4. [PubMed: 25620092]

40. Venkataraman D, et al., Filaggrin loss-of-function mutations are associated with food allergy in childhood and adolescence. J Allergy Clin Immunol, 2014 134(4): p. 876-882 e4. [PubMed: 25174864]

41. Akinbami LJ, et al., Status of childhood asthma in the United States, 1980-2007. Pediatrics, 2009 123 Suppl 3: p. S131-45. [PubMed: 19221156] Suppl

42. Eggesbo M, et al., The prevalence of allergy to egg: a population-based study in young children. Allergy, 2001 56(5): p. 403-11. [PubMed: 11350303] 
43. Osterballe M, et al., The prevalence of food hypersensitivity in an unselected population of children and adults. Pediatr Allergy Immunol, 2005 16(7): p. 567-73. [PubMed: 16238581] 
A

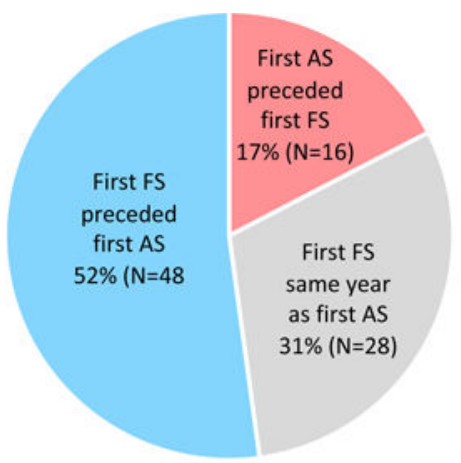

B

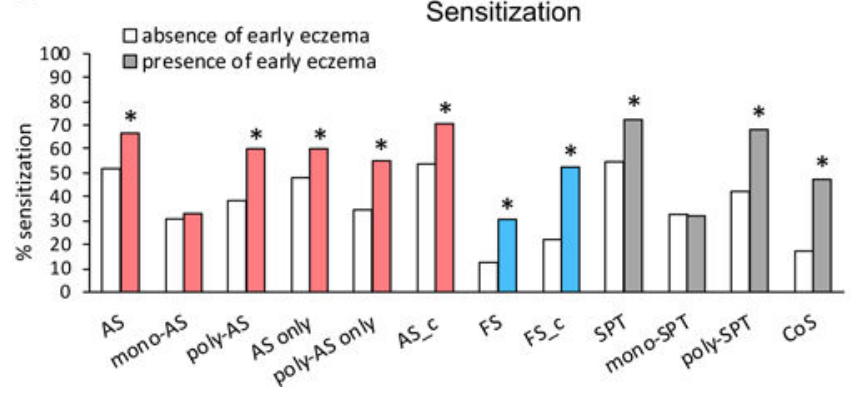

C

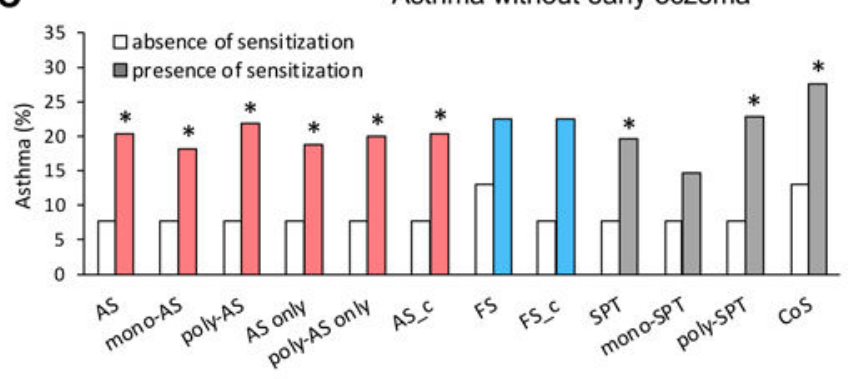

D

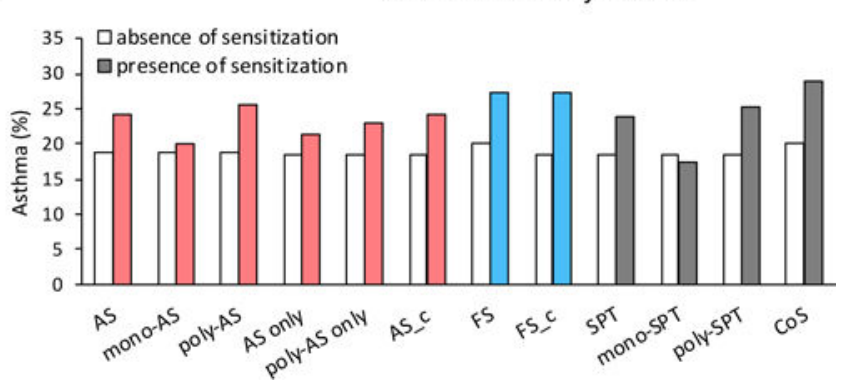

Figure 1.

Timing and patterns of sensitization, and their associations with asthma. A) Timing of first aerosensitization relative to first sensitization to food. B) Percentage of sensitization as a function of sensitization pattern, stratified for eczema. C) Percentage of asthma as a function of sensitization pattern in the absence of early eczema. D) Percentage of asthma as a function of sensitization pattern in the presence of early eczema. For B, C, and D, empty bars represents the control group for each sensitization pattern, and filled bars represent presence of the sensitization pattern. The control group for AS, mono-AS, and poly-AS was children without AS, the control group for FS was children without FS, and the control group for AS only, poly-AS only, AS_c, FS_c, SPT, mono-SPT, poly-SPT, and cosensitization was children without any positive SPT. An asterisk indicates a significant difference between filled bar and empty bar for a specific sensitization pattern. 

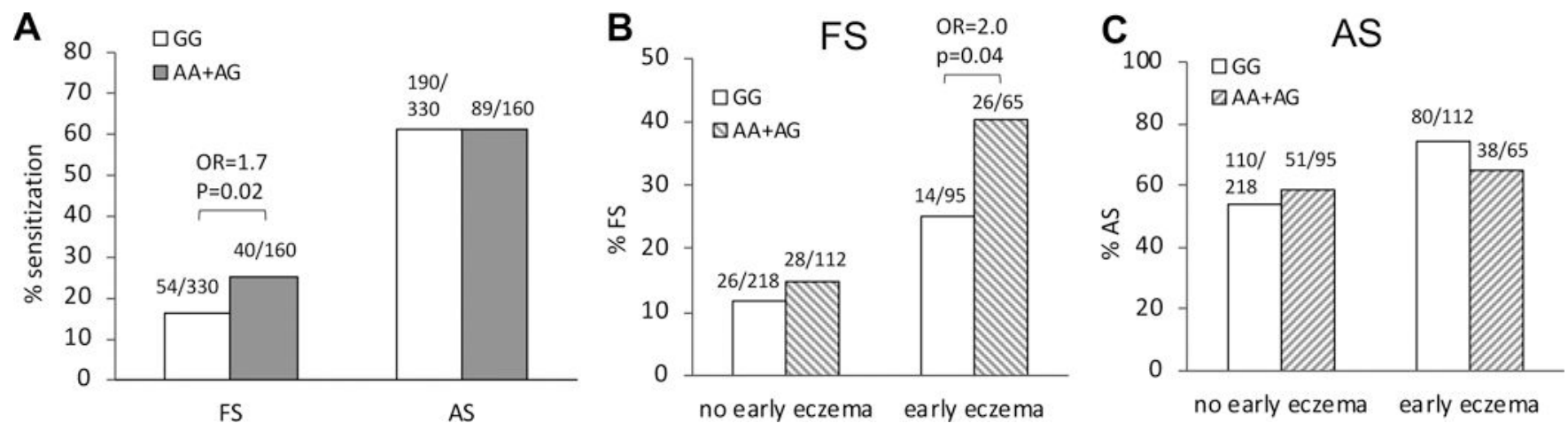

Figure 2.

A) Percentage of children sensitized to food vs. aeroallergens by age three in CCAAPS as a function of KIF3A rs 12186803 genotype. B) Percentage of children food-sensitized by age three in CCAAPS as a function of KIF3A rs 12186803 genotype, stratified for early eczema. C) Percentage of children aerosensitized by age three in CCAAPS as a function of $K I F 3 A$ rs 12186803 genotype, stratified for early eczema. P-values were determined using Fisher's exact test. 

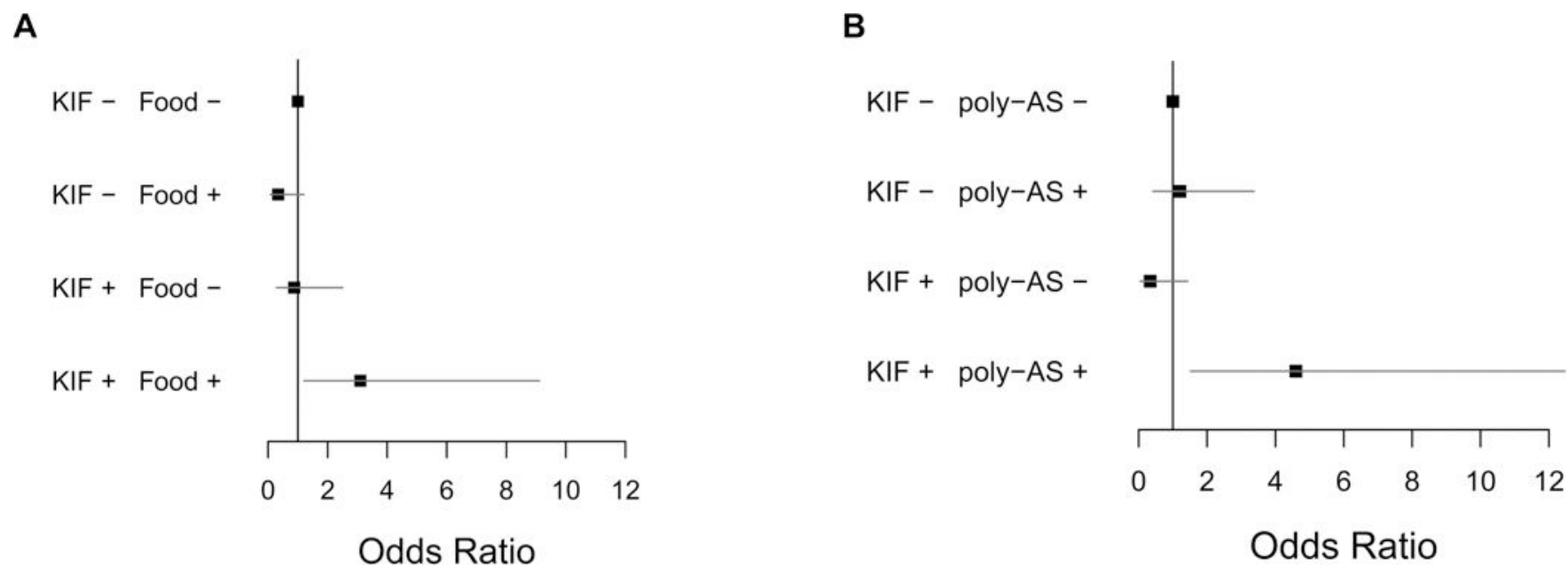

Figure 3.

A. Odds ratios for asthma risk in children with early eczema. The odds ratios were adjusted for early wheezing and parental asthma. The group of children having neither the KIF3A rs 12186803 risk genotype nor FS was used as reference. B. Odds ratios for asthma risk in children without early eczema. The odds ratios were adjusted for early wheezing and race. The group of children having neither the KIF3A rs 12186803 risk genotype nor FS was used as reference. 

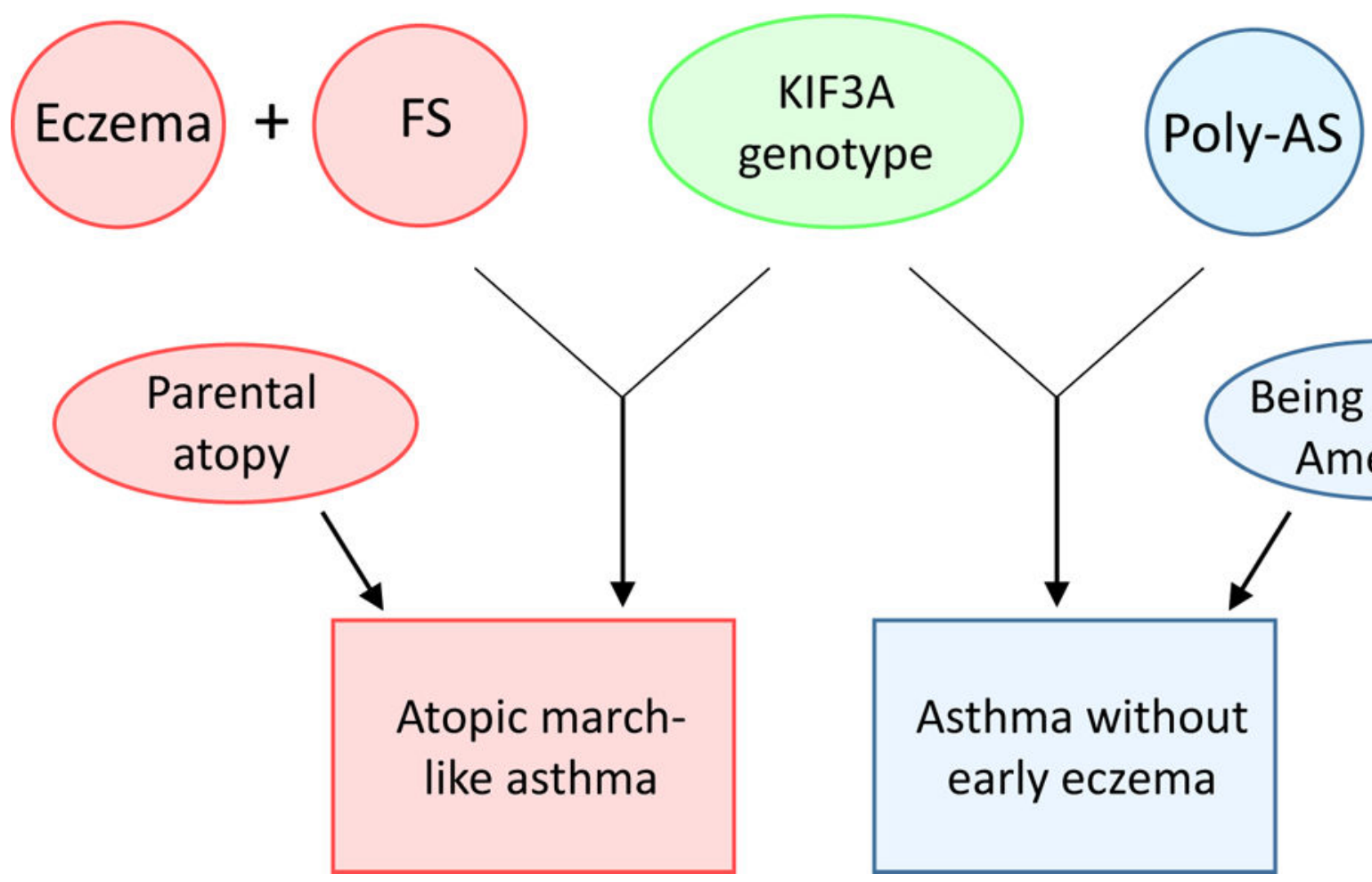

Figure 4.

Schematic summary of the predictors of the two groups at high risk for asthma. 
Table 1.

Definitions of sensitization variables

\begin{tabular}{|c|c|c|}
\hline Variable & Sensitization type & $\begin{array}{c}\text { Number of } \\
\text { sensitizations }\end{array}$ \\
\hline$A S^{a}$ & AS & $\geq 1$ \\
\hline mono- $A S^{a}$ & AS & 1 \\
\hline poly- $A S^{a}$ & AS & $\geq 2$ \\
\hline AS only ${ }^{c}$ & AS without FS & $\geq 1$ \\
\hline poly-AS only ${ }^{c}$ & AS without FS & $\geq 2$ \\
\hline$A S_{-} c^{c}$ & AS & $\geq 1$ \\
\hline$F S^{b}$ & FS & $\geq 1$ \\
\hline$F S_{-} c^{c}$ & FS & $\geq 1$ \\
\hline$S P T^{\mathcal{C}}$ & AS and/or FS & $\geq 1$ \\
\hline mono-SPT ${ }^{\mathcal{C}}$ & AS or FS & 1 \\
\hline poly-SPT $T^{\mathcal{C}}$ & AS and/or FS & $\geq 2$ \\
\hline $\operatorname{CoS}^{\mathcal{c}}$ & AS and FS & $\geq 2$ \\
\hline \multicolumn{3}{|c|}{ control group: AS negative; } \\
\hline$b$ control group: $F$ & negative; & \\
\hline
\end{tabular}

AS: aeroallergen sensitization; FS: food sensitization; CoS: cosensitization to food and aeroallergens; SPT: skin prick test. 
Table 2.

Demographic and clinical characteristics of the genotyped study cohort. P-values were obtained by chisquared test.

\begin{tabular}{lcccc}
\hline & $\begin{array}{c}\text { Total } \\
(\mathbf{N}=490)\end{array}$ & $\begin{array}{c}\text { No asthma } \\
(\mathbf{N = 4 0 5})\end{array}$ & $\begin{array}{c}\text { Asthma } \\
(\mathbf{N = 8 5} ; \mathbf{1 7 \%})\end{array}$ & $\mathbf{p}$ \\
\hline Male (\%) & 56 & 54 & 62 & 0.2 \\
African American (\%) & 21 & 17 & 38 & $<0.001$ \\
Parental asthma (\%) & 41 & 38 & 55 & 0.004 \\
Early wheeze (\%) & 18 & 12 & 46 & $<0.001$ \\
Early eczema (\%) & 36 & 34 & 47 & 0.03 \\
KIF3A rs12186803 risk allele (\%) & 33 & 31 & 42 & 0.049 \\
\hline
\end{tabular}




\section{를 \\ }

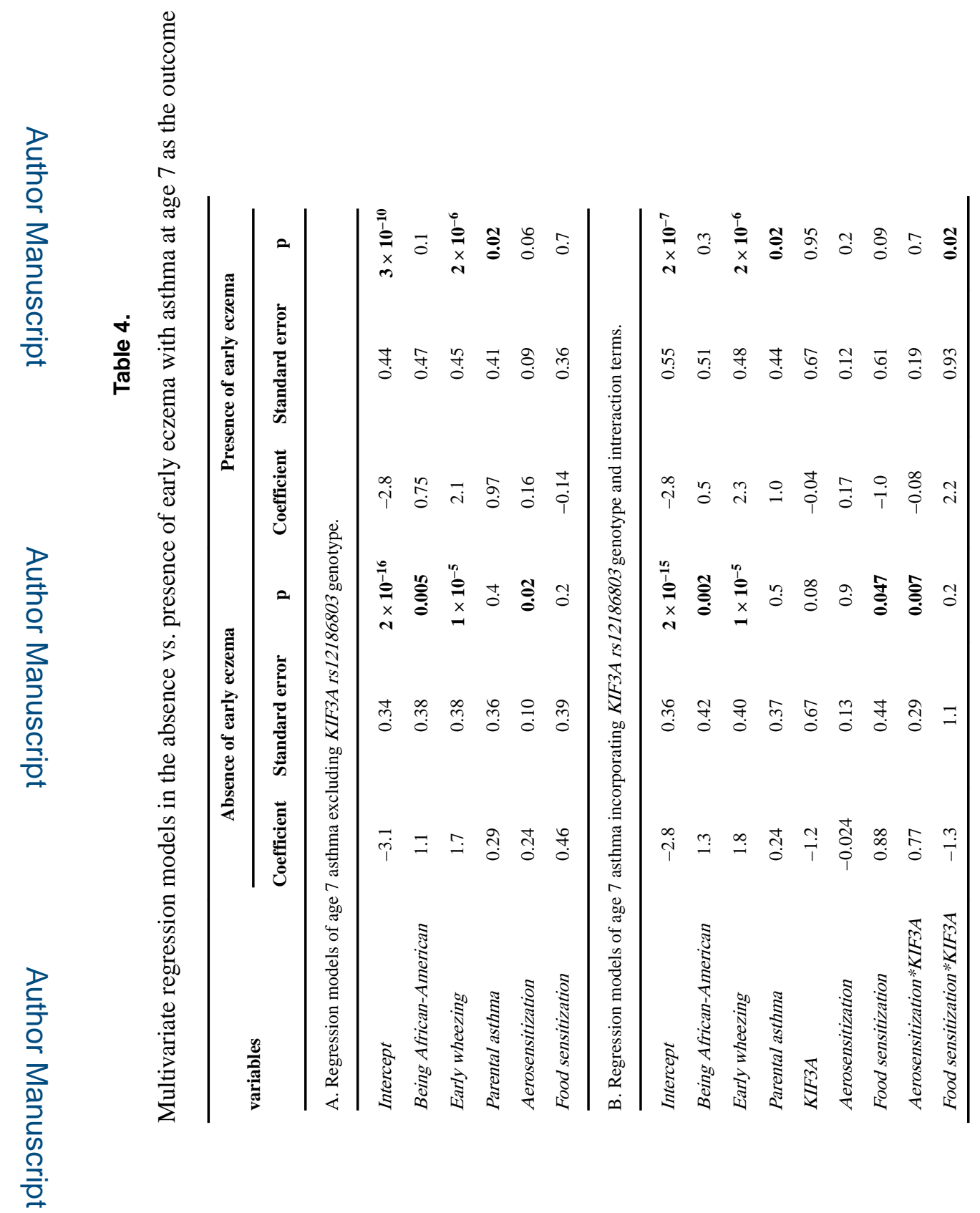

Clin Exp Allergy. Author manuscript; available in PMC 2020 June 01. 